Groups Geom. Dyn. 4 (2010), 533-548

DOI $10.4171 / \mathrm{GGD} / 94$
Groups, Geometry, and Dynamics

(C) European Mathematical Society

\title{
Codimension one subgroups and boundaries of hyperbolic groups
}

\author{
Thomas Delzant and Panos Papasoglu*
}

\begin{abstract}
We construct hyperbolic groups with the following properties: The boundary of the group has big dimension, it is separated by a Cantor set, and the group does not split. This shows that Bowditch's theorem that characterizes splittings of hyperbolic groups over 2-ended groups in terms of the boundary cannot be extended to splittings over more complicated subgroups.
\end{abstract}

Mathematics Subject Classification (2010). 20E08, 20F65, 20 F67.

Keywords. Boundary, hyperbolic group, splittings.

\section{Introduction}

Let $G$ be a finitely generated group and let $H$ be a subgroup of $G$. We say that $H$ is a codimension 1 subgroup if for some finite index subgroup $H^{\prime}$ of $H C_{G} / H^{\prime}$ has more than 1 end, where $C_{G}$ is the Cayley graph of $G$. If $G$ splits over $H$ then one easily sees that $H$ is codimension 1 . The converse is not true; for example, any closed geodesic on a surface group gives a cyclic codimension 1 subgroup of the fundamental group of the surface. On the other hand only simple closed geodesics correspond to splittings.

The surface example can be generalized to CAT(0) complexes to produce examples of codimension 1 subgroups: If $X$ is a finite CAT(0) complex of (say) dimension 2 and if $R$ is a locally geodesic track on $X$, then the subgroup of $G=\pi_{1}(X)$ corresponding to $R$ is a codimension 1 free subgroup of $G$. Wise ([11]) exploited this idea producing codimension 1 subgroups for small cancellation groups. In the setting of small cancellation groups of course one needs some combinatorial analog for the convexity property of geodesics (or tracks), and Wise develops such a notion. Pride ([9]) has shown that there are small cancellation groups that have property (FA) (see [10], p. 58), so such groups have codimension 1 subgroups but do not split.

Stallings showed that if a compact set separates the Cayley graph of a finitely generated group $G$ into at least two unbounded components, then $G$ splits over a

\footnotetext{
*We acknowledge support from the French-Greek grant Plato.
} 
finite group. Bowditch ([1]) showed something similar for hyperbolic groups: If the boundary $\partial G$ of a 1-ended hyperbolic group $G$ has a local cut point, then the group splits over a 2-ended group, unless it is a triangle group. There have been other generalizations of Stallings theorem similar in spirit. The general idea is that if a 'small' set (coarsely) separates the Cayley graph of a group, then the group splits over a subgroup quasi-isometric to the 'small set'. For a precise conjecture see [7].

The main purpose of this paper is to show some limitations of this 'philosophy'. Given any $n>0$, we produce an example of a hyperbolic group $G$ such that $\operatorname{dim}(\partial G)>n, \partial G$ is separated by a set of dimension 0 (a Cantor set) and $G$ has property (FA) (so it does not split over any subgroup). Our example is based on Wise's construction which we generalize to the setting of small cancellation theory over free products.

We would like to thank the referee for many suggestions and corrections that improved the exposition.

\section{Preliminaries}

Definition 1. A diagram is a finite connected planar graph. The faces of a diagram $D$ are the closures of the bounded components of $\mathbb{R}^{2}-D$.

In what follows we assume always that each interior vertex (i.e., not on $\partial D$ ) of a diagram has degree at least 3 . We can always achieve this by ignoring all interior vertices of degree 2 .

We will need some small cancellation results about diagrams shown by McCammond and Wise in [5]. For the reader's convenience and also because our setting is slightly different we include these results here. These results strengthen classical small cancellation results (see e.g. [4]).

We need some notation: If $D$ is a diagram we denote by $\partial D$ the boundary of the unbounded component of $\mathbb{R}^{2}-D$ (so if $U$ is the unbounded component of $\mathbb{R}^{2}-D$, $\partial D=\bar{U}-U)$. We say that the diagram is non-singular if $\partial D$ is homeomorphic to $S^{1}$. We say that an edge of $D$ is interior if it does not lie in $\partial D$.

If $D$ is a diagram we denote by $E, F, V$ respectively the total number of edges, faces and vertices of the diagram.

We denote by $E^{\bullet}, E^{\circ}$ respectively the number of edges of the diagram that lie (do not lie) in $\partial D$. We denote by $V^{+}$the number of vertices in $\partial D$ that lie in exactly one face and by $V^{-}$the number of vertices in $\partial D$ that lie in more than one face. We denote by $V^{\circ}$ the number of vertices of $D$ that do not lie on $\partial D$.

If $f$ is a face of $D$ then we can represent its boundary by a cycle $\left(e_{1}, \ldots, e_{n}\right)$, where the $e_{i}$ 's are oriented edges. We say that a diagram satisfies the $C(6)$ condition if the boundary cycle of each face of the diagram has at least 6 edges. We have the following version of Greedlinger's lemma (see [4]): 
Lemma 2.1. Let $D$ be a non-singular diagram that satisfies the condition $C(6)$. Then $V^{+} \geq V^{-}+6$.

Proof. We have the following inequalities. First,

$$
6 F \leq 2 E^{\circ}+E^{\bullet} .
$$

This is because each face has at least 6 edges and each interior edge appears twice as an edge of a face while each boundary edge appears once. Second,

$$
2 E \geq 3 V^{\circ}+3 V^{-}+2 V^{+} .
$$

This is because each edge yields 2 vertices and each interior vertex has degree at least 3 .

Finally, using Euler's formula, inequality (1) and the equality $E=E^{\circ}+E^{\bullet}$ we obtain

$$
V=E-F+1 \geq E-\frac{E^{\circ}}{3}-\frac{E^{\bullet}}{6}+1=\frac{2 E}{3}+\frac{E^{\bullet}}{6}+1 .
$$

We note that

$$
E^{\bullet}=V^{-}+V^{+} \text {. }
$$

Substituting $E^{\bullet}$ in (3) and using inequality (2) for $E$ we obtain

$$
V \geq \frac{2}{3}\left(\frac{3}{2} V^{\circ}+\frac{3}{2} V^{-}+V^{+}\right)+\frac{V^{-}+V^{+}}{6}+1 .
$$

Since

$$
V=V^{-}+V^{+}+V^{\circ}
$$

we obtain

$$
V^{+} \geq V^{-}+6 .
$$

We recall some definitions from [5]:

Definition 2. Let $D$ be a non-singular diagram. A face $F$ of the diagram is called an $i$-shell if the intersection of $F$ with the boundary of $D$ is connected and exactly $i$ edges of $F$ are interior edges of $D$.

Definition 3. We say that a diagram $D$ is a ladder if there are at most two faces $F_{1}, F_{2}$ of $D$ such that $D-F_{1}, D-F_{2}$ are connected while for every other face $F$ of $D, D-F$ has exactly 2 components, and for every vertex $v D-v$ has at most 2 components.

We have the following corollary from Lemma 2.1.

Corollary 2.2. Let $D$ be a non-singular disc diagram which is $C(6)$ and which contains no 3-shells and at most two $i$-shells with $i \leq 2$. Then either $D$ has a single face or it contains exactly two $i$-shells with $i \leq 2$ and it is a ladder. 


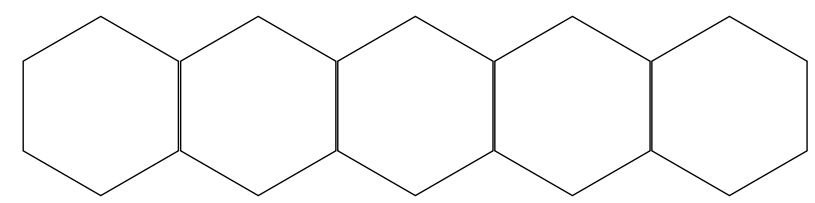

Figure 1. A ladder.

Proof. We modify $D$ as follows: if a face $F$ of $D$ has more than 6 edges and it intersects the boundary we ignore successively vertices of $F$ that do not lie on any other face till $F$ has 6 edges (or there are no more vertices to erase). Let us call $D_{1}$ the new diagram. $D_{1}$ is still a $C(6)$ diagram. $D_{1}$ contains also no 3 -shells and at most two $i$-shells for $i \leq 2$. We consider now a face $F$ of $D_{1}$ that intersects the boundary and we see how it contributes to $V^{+}, V^{-}$. If $F$ is not an $i$-shell then 2 vertices of $F$ contribute to $V^{+}$, while at least 4 vertices of $F$ contribute to $V^{-}$. So the total contribution of all such faces to the difference $V^{+}-V^{-}$is at most 0 (note that the contribution is not necessarily negative since we count twice the $V^{-}$vertices as they lie in at least 2 faces). The contribution of an $i$-shell to the difference $V^{+}-V^{-}$ is $4-i$.

Since $D$ contains no 3 -shells and at most $2 i$-shells for $i \leq 2$, the inequality

$$
V^{+}-V^{-} \geq 6
$$

implies that if $D_{1}$ has more than one face, then $D_{1}$ has exactly two 1-shells, say $F_{1}, F_{2}$. If we erase $F_{1}$ we obtain a diagram $D_{2}$ which is again $C(6)$. We note that $F_{1}$ intersects exactly one face of $D_{1}$, so after erasing it the diagram $D_{2}$ has still the other 1-shell of $D_{1}$ and at most one new $i$-shell for some $i \leq 3$. By the inequality $V^{+} \geq V^{-}+6$ again we conclude as before that either $D_{2}$ has only one face or it has exactly two 1 -shells $F_{2}, F_{3}$. Inductively we see that $D_{1}$ is a ladder hence $D$ is also a ladder.

We will need a more technical result. If $v$ is a vertex in a diagram we denote by $\mathrm{d}_{v}$ the degree of $v$. The result below will be used to show that small cancellation products of word hyperbolic groups are word hyperbolic.

Lemma 2.3. Let $D$ be a non-singular diagram that satisfies the condition $C(7)$. Then

$$
\frac{1}{3} \sum_{v \in D^{0}} \frac{\mathrm{d}_{v}}{2}-\frac{2 E^{\circ}}{7} \leq V^{\bullet}+\frac{E^{\bullet}}{7} .
$$

In particular

$$
F \leq 3 E^{\bullet}+3 V^{\bullet},
$$

i.e., D satisfies a linear isoperimetric inequality. 
Proof. We denote by $D^{0}$ the set of vertices of $D$ (the 0 -skeleton). Clearly

$$
\sum_{v \in D^{0}} \frac{\mathrm{d}_{v}}{2}=E
$$

We also have the inequality

$$
7 F \leq 2 E^{\circ}+E^{\bullet} .
$$

This is because each face has at least 7 edges and each interior edge lies in at most 2 faces while boundary edges lie in one face.

Using Euler's formula and the inequality above we obtain

$$
E+1=V+F \leq V^{\bullet}+\frac{E^{\bullet}}{7}+V^{\circ}+\frac{2 E^{\circ}}{7} .
$$

Since $\mathrm{d}_{v} \geq 3$ for every $v$ in the interior of $D$, it follows that

$$
\sum_{v \in D^{0}} \frac{\mathrm{d}_{v}}{2}-V^{\circ} \geq \frac{1}{3} \sum_{v \in D^{0}} \frac{\mathrm{d}_{v}}{2} .
$$

By (4) and (5) we have

$$
\frac{1}{3} \sum_{v \in D^{0}} \frac{\mathrm{d}_{v}}{2}-\frac{2 E^{\circ}}{7} \leq V^{\bullet}+\frac{E^{\bullet}}{7} .
$$

Since

$$
\frac{1}{3} \sum_{v \in D^{0}} \frac{\mathrm{d}_{v}}{2} \geq \frac{E^{\circ}}{3}
$$

we have

$$
\frac{1}{3} \sum_{v \in D^{0}} \frac{\mathrm{d}_{v}}{2}-\frac{2 E^{\circ}}{7} \geq \frac{E^{\circ}}{42} \geq \frac{3 F}{7}-\frac{E^{\bullet}}{84}
$$

and, using (6),

$$
V^{\bullet}+\frac{2 E^{\bullet}}{7} \geq \frac{3 F}{7}-\frac{E^{\bullet}}{84}
$$

which implies that $F \leq 3 E^{\bullet}+3 V^{\bullet}$.

\section{Small cancellation theory over free products}

Small cancellation theory can be developed over free products (see [4]). We show in this section that small cancellation products have codimension 1 subgroups. This generalizes a result of Wise ([11]). We recall that the free product factors embed in small cancellation products ([4], Corollary 9.4, p. 278). Osin ([6], Lemma 4.4) showed that free product factors embed quasi-isometrically in small cancellation products (this also follows from [2]). For the reader's convenience we include a proof of this below. 
Definition 4. Let $\langle S \mid R\rangle$ be a presentation of a group $G$. Here we consider $R$ to be a sequence of elements rather than a set. We say that $\langle S \mid R\rangle$ is symmetrized if every $r \in R$ is cyclically reduced word and for any $r=y_{1} \ldots y_{n} \in R, R$ contains $2 n$ distinct terms corresponding to the $2 n$ cyclic permutations of $r$ and $r^{-1}$. If $r_{1}=c b$, $r_{2}=c a$ and the words $c b, c a$ are reduced we call $c$ a piece of the presentation.

For example the symmetrized presentation that corresponds to the presentation $\left\langle a \mid a^{2}\right\rangle$ is the presentation $\left\langle a \mid a^{2}, a^{2}, a^{-2}, a^{-2}\right\rangle$.

Let now $\langle S \mid R\rangle$ be a symmetrized presentation. We have then the following small cancellation conditions:

- Condition $C^{\prime}(\lambda)$ : If $r \in R$ and $r=c b$ with $c b$ reduced word and $c$ a piece then $|c|<\lambda|r|$.

- Condition $C(p)$ : No element of $R$ is a product of fewer than $p$ pieces.

- Condition B(2p): If $r=a b$ and $a$ is a product of $p$ pieces then $|a| \leq|r| / 2$.

Wise showed in [11] that groups that admit a presentation in which all relators have even length and condition $B(6)$ is satisfied, have codimension 1 subgroups. Clearly condition $C^{\prime}(1 / 6)$ is stronger than condition $B(6)$, so Wise's result holds for these groups too.

We now describe the small cancellation conditions on free products ([4], ChapterV, Section 9). Let $F$ be the free product of the groups $A_{i}$.

A word in the free product $* A_{i}$ is a sequence of elements $a_{1}, \ldots, a_{n}$ where each $a_{i}$ is an element of a free factor.

We say that a word $a_{1} \ldots a_{n}$ is reduced if each $a_{j}$ is an element of one of the $A_{i}$ and $a_{j}, a_{j+1}$ belong to different factors for any $j$. Any element $g \in F$ can be represented in a unique way as a reduced word (normal form of $g$ ). If $g=a_{1} \ldots a_{n}$ is the normal form of $g$ we define $\|g\|=n$. If $u=a_{1} \ldots a_{n}, v=b_{1} \ldots b_{k}$ are reduced words we say that the word $u v=a_{1} \ldots a_{n} b_{1} \ldots b_{k}$ is semi-reduced if $a_{n} b_{1} \neq e$. Note however that $a_{n}, b_{1}$ might lie in the same factor. We say that a word $w=a_{1} \ldots a_{n}$ is weakly cyclically reduced if it is reduced and $a_{n} a_{1} \neq e$. We say that a sequence of words $R$ is symmetrized if whenever $r \in R$ all weakly cyclically reduced conjugates of $r$ and $r^{-1}$ are in $R$. We remark here that if the $A_{i}$ 's are infinite groups a symmetrized set of relations is infinite if it has length greater than 1 .

We say that $c$ is a piece if there are distinct $r_{1}, r_{2} \in R$ such that $r_{1}=c a, r_{2}=c b$ and the words $c a, c b$ are semi-reduced. As before we have the condition $C^{\prime}(\lambda)$ :

Condition $C^{\prime}(\lambda)$ : If $r \in R$ and $r=c b$ with $c b$ a semi-reduced word and $c$ a piece then $\|c\|<\lambda\|r\|$.

Let now $F$ be a free product $F=* A_{i}$ of non-trivial groups and let $R$ be a symmetrized subset of $F$. The group $G$ defined by the free product presentation $\langle F \mid R\rangle$ is the quotient

$$
G=F /\langle\langle R\rangle
$$

where $\langle\langle R\rangle\rangle$ is the normal closure of $R$ in $F$. 
We show that groups $G$ with free product presentations $\langle F \mid R\rangle$ that satisfy the $C^{\prime}(1 / 6)$ condition have codimension 1 subgroups. We start first by considering van Kampen diagrams over $G$. We consider a usual presentation of $G$ with a set of generators $S$ given by the generators of $A_{i}$ 's and a set of relators consisting of relators of the $A_{i}$ 's together with a set $R^{\prime}$ such that $R$ is obtained by taking all weak cyclic conjugates of elements in $R^{\prime}$ and their inverses. If $R^{\prime}$ is finite we say that $G$ has a finite free product presentation. Let now $w$ be a word in $S$ representing the identity in $G$. Let $D$ be a reduced van Kampen diagram for $w$ over the presentation given above. We remark that if $p$ is a simple closed path in the 1-skeleton of $D$ such that all edges of $p$ lie in a single factor $A_{i}$ then the word corresponding to $p$ represents the identity in $A_{i}$ (see [4], Corollary 9.4). Call such a simple closed path maximal if there is no other such simple closed path $q$ in the interior of $p$. We modify now the diagram $D$ as follows: For each maximal simple closed path $p$ we erase all edges of $p$ and all edges of $D$ inside $p$ and we introduce a new vertex $v_{p}$ which we join with all vertices of $p$. Now each edge $e$ of $p$ has been replaced by two edges $e_{1}, e_{2}$. We label $e_{1}, e_{2}$ by elements of $A_{i}$ so that the product of their labels is equal to the label of $e$. Note that we may label an edge by the identity. After this operation some of the edges of $D$ are 'subdivided'. We subdivide the remaining edges of $D$ so that the labels of the new edges lie in the same factor as the old ones and the product of their labels is equal to the label of the old edge. We call this a van Kampen diagram over the free product.

We remark now that the $C^{\prime}(\lambda)$ condition holds for this new diagram, i.e., if $p$ is a path in the diagram, which is contained in the intersection of two faces $R_{1} \cap R_{2}$ and intersects no other face, except possibly at its endpoints, then

$$
\text { length }(p)<\lambda \min \left(\text { length }\left(\partial R_{1}\right) \text {, length }\left(\partial R_{2}\right)\right) \text {. }
$$

We will show that groups that satisfy the small cancellation condition $C^{\prime}(1 / 6)$ over free products have codimension 1 free subgroups. The proof is similar to the proof in [11]. To simplify notation we show this below only in the case of free products with only two factors but the same proof applies in general.

Theorem 3.1. Let $G$ be a finitely generated group with a finite free product $C^{\prime}(1 / 6)$ presentation $\left\langle F \mid R^{\prime}\right\rangle$, where $F=A * B$. Assume further that each $r \in R^{\prime}$ is a cyclically reduced word and $\|r\|$ is even. Then $G$ has a codimension 1 subgroup.

Proof. We construct a complex for $G$ as usual. If $K_{1}, K_{2}$ are complexes with a single vertex respectively $x_{1}, x_{2}$ such that $\pi_{1}\left(K_{1}, x_{1}\right)=A, \pi_{1}\left(K_{2}, x_{2}\right)=B$ we take the wedge product $K_{1} \vee K_{2}$ of identifying $x_{1}, x_{2}$ to a single vertex $x$. For each $r \in R^{\prime}$ we glue a 2-cell, $c(r)$, to $K_{1} \vee K_{2}$ in the obvious way to obtain a complex $K$ such that $\pi_{1}(K, x)=G$. We argue now in a way similar to Wise ([11]). We slightly change the approach and we consider bouquets of circles that go through $x$ rather than tracks. We explain now how to construct a bouquet of circles $\Gamma$ which will generate a codimension 1 subgroup $H$. Algebraically $H$ is the subgroup generated 
by all 'halves' of relators in $R^{\prime}$. So if $r \in R^{\prime}$ and $r=c_{1} c_{2} \ldots c_{2 n}$ is the normal form of $r$ in $A * B$ then $c_{1} c_{2} \ldots c_{n}$ is a generator of $H$. We take also all cyclic permutations of the normal form of $r: c_{2} \ldots c_{2 n} c_{1}, c_{3} \ldots c_{2 n} c_{1} c_{2}, \ldots$, and we include the halves of these relations to the generating set of $H$.

Let $r \in R^{\prime}$ and let $r=a_{1} b_{1} \ldots a_{n} b_{n}$ be the normal form of $r$ in $F$. We represent the 2-cell $c(r)$ corresponding to $r$ as a polygon, where the $a_{i}, b_{i}$ 's are the labels of the sides of this polygon. We can represent the generators of $H$ geometrically as 'diagonals' of the cells $c(r)\left(r \in R^{\prime}\right)$. We remark now that since $c(r)$ has an even number of sides each vertex has a vertex opposite to it, so for each vertex there is a diagonal joining it with the opposite vertex. Note that this is slightly different from the way one usually represents $r$ as a polygon, where the sides of the polygon correspond to the letters of $r$. In our context, however, we ignore most of these vertices and we keep only the ones that correspond to the normal form representation of $r$ in the free product $A * B$. So if we see $r$ in the usual way as a polygon, where the sides are letters, then, in our terminology, some vertices have opposite vertices, while others do not.

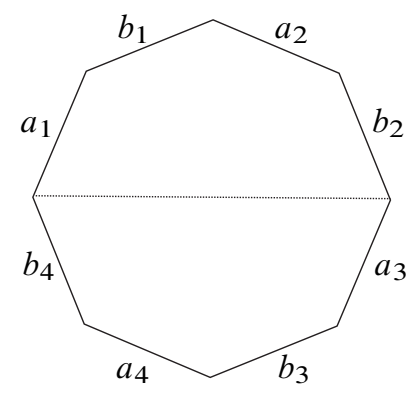

Figure 2. A generator (represented by a dotted line).

We will define now $\Gamma$ as an abstract graph. $\Gamma$ has a single vertex and the edges of $\Gamma$ correspond to diagonals of the cells $c(r)\left(r \in R^{\prime}\right)$.

We remark that $\Gamma$ is a bouquet of circles if looked at as an abstract graph, but if we see it as immersed in $K$, some of its edges intersect each other in the middle points of the polygons.

If we see $\Gamma$ as a subset of $K$, its edges (loops) generate $H$.

We will show that $H$ is a codimension 1 subgroup of $G$.

Lemma 3.2. There is a tree $\widetilde{\Gamma} \subset \widetilde{K}$ whose edges are diagonals of 2-cells such that $\widetilde{\Gamma}$ is invariant under $H . \widetilde{K}-\widetilde{\Gamma}$ has at least 2 components.

Proof. We will show that the universal covering of $\Gamma$ embeds in $\widetilde{K}$ as a graph $\widetilde{\Gamma}$. The graph $\widetilde{\Gamma}$ projects under the covering projection $\pi: \widetilde{K} \rightarrow K$ to the image of $\Gamma$ in $K$.

Let $v \in \widetilde{K}$ be a vertex. We define a connected graph in $\widetilde{K}$ as follows: We say that two vertices are related if they are opposite. We take the equivalence relation 
generated by this relation and we consider the equivalence class of $v$. Let $\widetilde{\Gamma}$ be the graph obtained by joining opposite vertices in this equivalence class by diagonals. We claim that $\widetilde{\Gamma}$ is a tree. If it is not a tree then there is a path $p$ in $\widetilde{\Gamma}$ such that both endpoints of $p$ lie on the same 2-cell of $R^{\prime}$ and $p$ is not a single edge (a diagonal). Let us say that $a, b$ are the endpoints of $p$ and they lie on a 2-cell $\sigma$. Let $q$ be a path on $\partial \sigma$ joining $a, b$. We may assume $q$ to have minimal normal form length in the free product among the 2 possible paths. Now $p \cup q$ is a closed loop. We change now $p$ by replacing each diagonal with the corresponding path on the boundary on which the diagonal lies. We note that we have two choices and we replace the diagonals so that the path we obtain by replacing all of them corresponds to a reduced word of $F$. Let $p^{\prime}$ be the path we obtain in this way. We may arrange also that $p^{\prime} \cup q$ is reduced at the vertex $a$ (unless $a=b$ ). We now consider the van Kampen diagram over the free product for $p^{\prime} \cup q$ and observe that if it has an $i$-shell for $i \leq 2$, then the boundary of this $i$-shell contains a neighborhood of the vertex $b$. But this contradicts Corollary 2.2. It follows that $\widetilde{\Gamma}$ is a tree. By construction $\widetilde{\Gamma}$ is invariant under $H$ and separates locally (hence also globally) $\widetilde{K}$.

The first part of the next lemma also follows from work of Osin ([6], see also [2]). We include a proof here for the sake of completeness.

Lemma 3.3. The vertex groups $A, B$ and $H$ embed quasi-isometrically in $G$. Then $H$ is a codimension 1 subgroup of $G$.

Proof. Let $a$ be a geodesic word in the Cayley graph of $A$ (or $B$ ). We will show that $a$ is a quasi-geodesic in $\widetilde{K}$. Let $S$ be the generating set of $G$ and let $|w|$ be the length of a word in $S$. Put

$$
M=\max \left\{|r|: r \in R^{\prime}\right\} .
$$

If we think of $w$ as an element of $F=A * B$ rather than as a word in $S$ then we denote by $\|w\|$ its normal form length.

We define a new length function $L$ for words in $S$ :

$$
L(w)=M\|w\|+|w| .
$$

We refer to a path (word) of minimal $L$-length as an $L$-geodesic.

Obviously an $L$-geodesic is a quasi-geodesic.

We show now that $a$ is a quasi-geodesic. Let $p$ be an $L$-geodesic in the 1 -skeleton of $\widetilde{K}$ with the same endpoints as $a$. We consider the van Kampen diagram over the free product for $a \cup p$. We may assume that $a \cap p$ is equal to the endpoints of $a, p$ since along the intersection of $a, p$, the path $a$ is quasi-geodesic (this is because $p$ is a quasi-geodesic).

This diagram has at most $2 i$-shells (with $i \leq 2$ ), because by the definition of $L$-geodesics it follows that an $i$-shell with $i=1$ or 2 contains at least one endpoint of $p$. Thus by Corollary 2.2 this diagram is a ladder. By considering now the usual van Kampen diagram for $a \cup p$ we have that $|a| \leq M|p|$ so $a$ is quasi-geodesic. 
We prove now that $H$ is quasi-isometrically embedded. Since $H$ acts freely on $\widetilde{\Gamma}$ it is enough to show that $\widetilde{\Gamma}$ is quasi-isometrically embedded. Let $p$ be a geodesic path in $\widetilde{\Gamma}$ joining two vertices $v, u$ of $\widetilde{\Gamma}$.

We change $p$ by replacing each diagonal by a corresponding path on the boundary of the cell on which lies the diagonal. We pick this path so that the word of $F$ corresponding to the path is reduced (in the sense of normal forms). We note that this is possible since each diagonal can be replaced by two different paths, one starting with an $a$ and the other with a $b$.

Let $p^{\prime}$ be the path we obtain in this way. Let $q$ be an $L$-geodesic path joining $v, u$. As before we may assume that $p^{\prime}, q$ intersect only at their endpoints. Again by the definition of $p^{\prime}, q$ if we consider the van Kampen diagram over the free product for $p^{\prime} \cup q$ we remark that it has at most $2 i$-shells with $i \leq 2$.

Hence this diagram is a ladder. By considering the usual van Kampen diagram we have that $\left|p^{\prime}\right| \leq M|q|$. Since $q$ is a quasi-geodesic we have that $p^{\prime}$ is a quasigeodesic, so $H$ is quasi-isometrically embedded.

Finally we show that $H$ is a codimension 1 subgroup. It suffices to show that $\widetilde{K}-\widetilde{\Gamma}$ has at least 2 components which are not contained in a finite neighborhood of $\widetilde{\Gamma}$. By its definition $\widetilde{\Gamma}$ separates locally $\widetilde{K}$. Since $\widetilde{K}$ is simply connected, $\widetilde{\Gamma}$ separates $\widetilde{K}$.

We introduce now some useful terminology. Let $r \in R^{\prime}$ and let $c_{1} c_{2} \ldots c_{n}$ be the normal form of $r$ in $F$. Recall that $r$ is cyclically reduced. Let $k$ be the smallest number such that

$$
k \geq \frac{n}{6} .
$$

We say then that $c_{1} \ldots c_{k}$ is a fragment of $r$. Similarly we define fragments of all cyclic permutations of $c_{1} c_{2} \ldots c_{n}$.

Let $R$ be a 2-cell in $\widetilde{K}$ which intersects $\widetilde{\Gamma}$ on an edge $e$. Let $v, u$ be the vertices of $e$.

Let $c_{1} c_{2} \ldots c_{n}$ be the label of $R$ starting from $v$ and written in free product normal form. Let $s$ be the vertex corresponding to the endpoint of a fragment $p_{1}=c_{1} \ldots c_{k}$ of $R$ starting at $v$. We construct a path starting from $v$ and lying in the same component of $\tilde{X}-\widetilde{\Gamma}$ as $s$. The path starts at $p_{1}$. At $s$ we continue by following $p_{1}$ with a fragment $p_{2}$ of another 2-cell $R_{2}$ corresponding to $r_{2} \in R^{\prime}$. We pick $R_{2} \neq R$ and so that $p_{1} p_{2}$ is reduced in $F$ (in the sense of normal forms). We continue inductively in the same way picking each time a new 2-cell and a fragment so that the word we obtain is reduced in $F$. This is clearly possible, since at each step, we consider a new 2-cell and we have two possibilities for going around the boundary of the cell (corresponding to the two orientations of the boundary). At least one of the two possibilities produces a path reduced in the sense of normal forms.

Let $\beta=p_{1} \ldots p_{n}$ be the path we obtain after $n$ steps. If $s_{n}$ is the endpoint of $p_{1} \ldots p_{n}$ we claim that $d\left(s_{n}, \widetilde{\Gamma}\right) \rightarrow \infty$ as $n \rightarrow \infty$. Indeed, let $q$ be a geodesic joining $s_{n}$ to a closest vertex $t \in \widetilde{\Gamma}$. We consider a geodesic $\gamma$ in $\widetilde{\Gamma}$ joining $v$ to $t$.

We distinguish two cases. Assume first that $u$ does not lie on $\gamma$. We change $\gamma$ by replacing each diagonal by the corresponding path on the boundary of the 2-cell in 


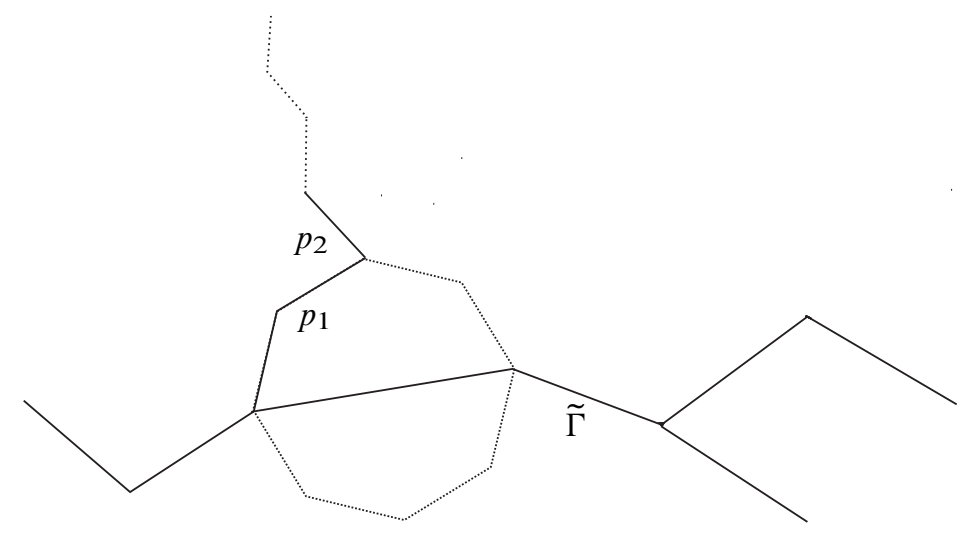

Figure 3. The tree $\widetilde{\Gamma}$ and the path $\beta=p_{1} p_{2} \ldots p_{n}$.

which the diagonal lies to obtain a path $\gamma^{\prime}$. We make these replacements so that the word of $F$ corresponding to the new path is reduced and $p_{1}^{-1} \gamma^{\prime}$ corresponds also to a reduced word in $F$. Clearly this is possible since we have two choices for replacing each diagonal and the normal form of each starts from a different free factor. We now consider the loop

$$
\beta \cup \gamma^{\prime} \cup q
$$

Since $q$ is geodesic, the van Kampen diagram for free products for this loop has at most $2 i$-shells with $i \leq 2$ and these would have to appear around the endpoints of $q$. It follows that this diagram is a ladder (see Corollary 2.2); hence the lengths of $q$ and $\beta \cup \gamma^{\prime}$ are comparable, so the length of $q$ goes to infinity as $n \rightarrow \infty$.

Now we deal with the second case, i.e., we assume that $u$ lies on $\gamma$. We modify $p_{1} \ldots p_{n}$ as follows. We replace $p_{1}$ by the path $q_{1}$ on the boundary of $R$ joining $s$ to $u$. We note that the new path $\beta^{\prime}=q_{1} p_{2} \ldots p_{n}$ might not be reduced at the endpoint of $q_{1}$. We replace $\gamma$ by a path $\gamma^{\prime}$ in the 1 -skeleton of $\widetilde{K}$ as before so that $q_{1}^{-1} \gamma^{\prime}$ is reduced in the free product $F$. We remark that the van Kampen diagram over free products for the loop

$$
\beta^{\prime} \cup \gamma^{\prime} \cup q
$$

is a ladder in this case too, hence the length of $q$ goes to infinity as $n \rightarrow \infty$.

Similarly we see that the component of $R-\widetilde{\Gamma}$ that does not contain $v$ is not contained in a finite neighborhood of $\widetilde{\Gamma}$. It follows that $H$ is codimension 1 .

This finishes the proof of Theorem 3.1. 


\section{The example}

Theorem 4.1. Given any $n>0$, there is a one-ended hyperbolic group $G$ such that

- $\operatorname{dim} \partial G \geq n$,

- $\partial G$ is separated by a Cantor set,

- $G$ does not split.

Proof. Let $A$ be a torsion free 1-ended hyperbolic group with property $T$ and such that $\operatorname{dim}(\partial A) \geq n$ (e.g., a lattice in $\operatorname{Sp}(n, 1))$. Let us denote $A=\left\langle a_{1}, \ldots, a_{k}\right\rangle$. We may assume that each $a_{i}$ is non-trivial and that $a_{i}^{m} \neq a_{j}^{r}$ for any $i \neq j$ and $m, r>0$. Let $B$ denote a copy of $A$ with corresponding generators $\left\langle b_{1}, \ldots, b_{k}\right\rangle$. We consider the free product $A * B$ and define $G$ to be the small cancellation quotient of $A * B$ given by the relations

$$
r_{i, j}=\left(a_{i} b_{j}\right)\left(a_{i} b_{j}^{2}\right) \ldots\left(a_{i} b_{j}^{19}\right), \quad 1 \leq i, j \leq k .
$$

By Theorem 3.1, $G$ has a free codimension 1 subgroup $H$. As shown in the proof of Theorem 3.1, $H$ is quasi-isometrically embedded, so a Cantor set separates $\partial G$.

We show now that $G$ has property (FA) (i.e., it does not split). Clearly $G$ is not an HNN extension since the abelianization of $A$ is finite, so the abelianization of $G$ is finite. We show that $G$ does not split as an amalgamated product. Suppose $G=X *_{C} Y$. Without loss of generality we may assume that $A \subset X$ and $B \subset g X g^{-1}$ or $B \subset g Y^{-1}$. Let $g=x_{1} \ldots x_{n}$ be the normal form of $g$ in the free product decomposition. By replacing $A, B$ by conjugates we may assume that either $g=1$ and $B \subset Y$, or $x_{1} \notin X$. However, we see then that the word

$$
r_{i, j}=\left(a_{i} b_{j}\right)\left(a_{i} b_{j}^{2}\right) \ldots\left(a_{i} b_{j}^{19}\right)
$$

is reduced in $X *_{C} Y$, unless $a_{i}$ or $b_{j}$ is in $C$. Since all $r_{i, j}$ are equal to the identity, this implies that $A=C$ and $B$ is contained in $Y$, or $B=C$ and $A$ is contained in $X$; but in both cases, the splitting would be trivial.

We claim finally that $G$ is hyperbolic. Indeed this follows by Lemma 4.4 of [6], and [2]. For the reader's convenience we sketch a proof here using Lemma 2.3. It is enough to show that $G$ satisfies a linear isoperimetric inequality. Let $w$ be a word on the generators of $G$ and let $D$ be a reduced van Kampen diagram for $G$. As described in Section 3, one obtains from $D$ a new diagram, say $D_{1}$, which is called the diagram for $w$ over the free product. Since $A, B$ are hyperbolic they satisfy some isoperimetric inequality of the form

$$
A(p) \leq K l(p)
$$

for any simple closed path $p$ in the Cayley graph of $A$ or of $B$.

It follows that if $p$ is a simple closed path of $D$ such that all edges of $p$ lie in $A$ (or in $B$ ) and if $v$ is the vertex of $D_{1}$ that we obtain by collapsing $p$ to a point, then

$$
\mathrm{d}_{v}=l(p) \geq \frac{1}{K} A(p),
$$


where $\mathrm{d}_{v}$ is the degree of $v$. It follows that

$$
A(D) \leq A\left(D_{1}\right)+K \sum_{v \in D_{1}^{0}} \mathrm{~d}_{v} .
$$

From Lemma 2.3 we have the following inequality for the diagram $D_{1}$ :

$$
\frac{1}{3} \sum_{v \in D_{1}^{0}} \frac{\mathrm{d}_{v}}{2}-\frac{2 E^{\circ}}{7} \leq V^{\bullet}+\frac{E^{\bullet}}{7} .
$$

Moreover,

$$
\sum_{v \in D_{1}^{0}} \frac{\mathrm{d}_{v}}{2} \geq E^{\circ} \Rightarrow \frac{2 E^{\circ}}{7} \leq \frac{2}{7} \sum_{v \in D_{1}^{0}} \frac{\mathrm{d}_{v}}{2},
$$

so

$$
\frac{1}{3} \sum_{v \in D_{1}^{0}} \frac{\mathrm{d}_{v}}{2}-\frac{2 E^{\circ}}{7} \geq \frac{1}{42} \sum_{v \in D_{1}^{0}} \frac{\mathrm{d}_{v}}{2} .
$$

We also have $l\left(\partial D_{1}\right) \leq l(\partial D)$ and $V^{\bullet}, E^{\bullet} \leq l(\partial D)$. Thus, by Lemma 2.3, it follows that

$$
A\left(D_{1}\right) \leq 6 l(\partial D)
$$

and

$$
\sum_{v \in D_{1}^{0}} \mathrm{~d}_{v} \leq 42 V^{\bullet}+7 E^{\bullet}
$$

hence

$$
A(D) \leq(6+49 K) l(\partial D) .
$$

In other words, $G$ satisfies a linear isoperimetric inequality, so it is hyperbolic.

Remark 1. The above example also shows that for any $n$ there is a finitely presented group $G$ with asdim $G>n$, which is separated coarsely by a uniformly embedded set $H$ of asdim $H=1$ and which does not split. This answers a question in [7].

\section{Boundaries and Cantor manifolds}

We recall some notions from dimension theory.

Definition 5. We say that a closed subset $F$ of a topological space $X$ separates $X$ if $X-F=U_{1} \cup U_{2}$ with $U_{1}, U_{2}$ open disjoint sets.

Definition 6. A topological space $X$ of dimension $n$ is a Cantor manifold if it cannot be separated by a closed sets $F$ such that $\operatorname{dim} F \leq n-2$. 
Hurewicz and Tumarkin showed the following:

Theorem 5.1. A compact metrizable topological space $X$ of dimension $n$ contains an n-dimensional Cantor manifold.

Definition 7. A maximal Cantor manifold of dimension $n$ of a compact metrizable topological space $X$ of dimension $n$ is called a dimensional component of $X$.

It is easy to see, using Zorn's lemma, that each Cantor manifold of $X$ is contained in a dimensional component and that dimensional components are closed.

It was conjectured in [7] that if the boundary of a hyperbolic group $G$ is not a Cantor manifold, then the group splits. This was motivated by a desire to generalize Stallings ends theorem to splittings over infinite groups. As we saw in the previous sections, this is false, but perhaps the group might split when the dimensional components themselves separate. So we have the following:

Question. Let $G$ be a hyperbolic group such that $\partial G$ is not a Cantor manifold and there is a dimensional component $C$ of $\partial G$ such that $\partial G-C$ is not connected. Is it true then that $G$ splits?

We remark that in the example presented in the previous section the dimensional components correspond to the boundaries (of conjugates of the subgroups $A, B$ ). So we have infinitely (countably) many distinct dimensional components, but $\partial G-C$ is connected for any dimensional component in this example.

Proposition 5.2. Let $G$ be a hyperbolic group such that $\partial G$ is not a Cantor manifold and there is a dimensional component $C$ of $\partial G$ such that $\partial G-C$ is not connected. Then $G$ acts non-trivially, by isometries, on an $\mathbb{R}$-tree $T$.

Proof. We give only a sketch since this type of argument is standard by now. Without loss of generality we assume that $\partial G$ is connected. Let $C$ be a dimensional component of $\partial G$ for which $\partial G-C$ is not connected. Let $R$ be a connected component of $\partial G-C$. Observe that

$$
\partial R=\bar{R}-R
$$

is not a single point since $\partial G$ has no cut points.

Let

$$
\mathcal{P}=\{g R: g \in G\} .
$$

Note that if $A_{1}, A_{2} \in \mathcal{P}$, then $A_{1} \subset A_{2}$ or $A_{2} \subset A_{1}$ or $A_{1} \cap A_{2}=\emptyset$.

We define now a betweeness relation in $\mathcal{P}$ that turns $\mathcal{P}$ into a pre-tree.

If $x, y, z$ are three distinct elements of $\mathcal{P}$, we say that $y$ is between $x$ and $z$ if one of the following four possibilities holds:

1. $z \subset y$ and $y \cap x=\emptyset$. 
2. $z \subset y$ and $y \subset x$.

3. $x \subset y$ and $y \cap z=\emptyset$.

4. $x \subset y$ and $y \subset z$.

If $y$ is between $x, z$ we write $x y z$.

We show now the axioms of pre-trees hold (see [8]):

1. By definition $x y x$ does not hold.

2. If $x y z$ holds then by the symmetry between $x, z$ in the definition above we see that $z y x$ holds as well.

3. If $x y z$ holds then by inspecting the four cases of the definition above we see that $x z y$ does not hold.

4. If $x y z$ holds and $w \neq y$ we will show that either $x y w$ or $z y w$.

Since $x y z$ holds, we may assume that $z \subset y$ (the case $x \subset y$ is treated similarly). We have now three cases: $y \subset w, w \subset y$ or $y \cap w=\emptyset$. If $y \subset w$ then $z y w$ holds. If $w \subset y$ then $x y w$ holds. Finally, if $y \cap w=\emptyset$ then $z y w$ holds.

By the standard 'join the dots' construction (see eg [8]) one can promote $\mathcal{P}$ to an $\mathbb{R}$-tree $T$. The action of $G$ on $\partial G$ induces an action of $G$ on $T$. It is easy to see that this action is non-nesting and that there is no fixed point. It follows [3] that $G$ acts by isometries on an $\mathbb{R}$ tree $T^{\prime}$. On the other hand it is not clear whether the action of $G$ on $T$ (hence also on $T^{\prime}$ ) is stable, so it is not clear whether $G$ splits.

One can easily construct continua with uncountably many dimensional components. However, when constructing hyperbolic groups using amalgamation or other standard constructions, the dimensional components correspond to quasi-isometrically embedded subgroups (so there are at most countably many such components). It is reasonable to ask:

Question. Let $G$ be a hyperbolic group. Is it true that $\partial G$ has at most countably many distinct dimensional components?

\section{References}

[1] B. H. Bowditch, Cut points and canonical splittings of hyperbolic groups. Acta Math. 180 (1998), 145-186. Zbl 0911.57001 MR 1638764

[2] T. Delzant, Sous-groupes distingués et quotients des groupes hyperboliques. Duke Math. J. 83 (1996), 661-682. Zbl 0852.20032 MR 1390660

[3] G. Levitt, Non-nesting actions on real trees. Bull. London Math. Soc. 30 (1998), 46-54. Zbl 0921.20031 MR 1479035

[4] R. C. Lyndon and P. E. Schupp, Combinatorial group theory. Ergeb. Math. Grenzgeb. 89, Springer-Verlag, Berlin 1977. Zbl 0368.20023 MR 0577064

[5] J. P. McCammond and D. T. Wise, Fans and ladders in small cancellation theory. Proc. London Math. Soc. (3) 84 (2002), 599-644. Zbl 1022.20012 MR 1888425 
[6] D. Osin, Small cancellations over relatively hyperbolic groups and embedding theorems. Ann. of Math., to appear. arXiv:math/0411039

[7] P. Papasoglu, Group splittings and asymptotic topology. J. Reine Angew. Math. 602 (2007), 1-16. Zbl 1160.20034 MR 2300450

[8] P. Papasoglu and E. Swenson, From continua to $\mathbb{R}$-trees. Algebr. Geom. Topol. 6 (2006), 1759-1784. Zbl 1182.54039 MR 2263049

[9] S. J. Pride, Some finitely presented groups of cohomological dimension two with property (FA). J. Pure Appl. Algebra 29 (1983), 167-168. Zbl 0513.20019 MR 707619

[10] J-P. Serre, Trees. Springer-Verlag, Berlin 1980. Zbl 0548.20018 MR 0607504

[11] D. T. Wise, Cubulating small cancellation groups. Geom. Funct. Anal. 14 (2004), 150-214. Zbl 1071.20038 MR 2053602

Received July 16, 2008; revised August 10, 2009

T. Delzant, Institut de Recherche Mathématique Avancée, Université de Stasbourg et CNRS, 7 rue René Descartes, 67084 Strasbourg Cedex, France

E-mail: delzant@math.u-strasbg.fr

P. Papasoglu, Mathematics Department, University of Athens, Panepistimioupolis, 15784 Athens, Greece

E-mail: panos@math.uoa.gr 